\title{
Cytarabine Monophosphate Prodrug MB07133
}

National Cancer Institute

\section{Source}

National Cancer Institute. Cytarabine Monophosphate Prodrug MB07133. NCI

Thesaurus. Code C128888.

A prodrug of the monophosphate (MP) form of the antimetabolite cytarabine (araCMP), an analogue of cytidine with a modified sugar moiety (arabinose instead of ribose), with potential antineoplastic activity. Upon administration of the cytarabine MP prodrug MB07133, the targeting moiety of this agent specifically delivers the cytarabine moiety to the liver. In turn, araCMP is selectively converted to araC triphosphate (araCTP) by a liver kinase, where it binds to and competes with cytidine for incorporation into DNA, thereby inhibiting DNA polymerase, and DNA synthesis. This leads to the inhibition of tumor cell proliferation and destruction of liver cancer cells. The liver is not able to convert araC into araCMP; araCMP is not converted into araCTP in tissues other than the liver. This enhances efficacy and minimizes systemic toxicity. 